\title{
Decreasing Low Acuity Pediatric Emergency Room Visits with Increased Clinic Access and Improved Parent Education
}

\author{
Toren Davis, DO, Albert Meyer, MD, Janalynn Beste, MD, and Sonali Batish, MPH
}

Background and Objective: The goal of this study was to decrease avoidable, low-acuity emergency department (ED) use among pediatric patients at Coastal Family Medicine. The rationale behind this focus was to improve continuity for our patients while decreasing the cost burden for low-acuity ED visits. The family medicine residency clinic pediatric panel has grown by $35 \%$ over the past 3 years, bringing this issue of same-day acute access in our clinic to the forefront.

Methods: A survey was created to better understand the needs of our high users of the ED. The survey identified that patients believed the ED provided better same-day access than our clinic during the daytime hours, 8 AM-5 PM, Monday-Friday. By using this data, along with a literature review and a community practice review, a business-hour walk-in clinic for ages 0 years to 18 years was started to improve access. Clinic posters, revised scripting for office staff, phone room staff, and our after-hour triage line as well as bookmarks advertising the walk-in clinic given during well-child checks were created to address parent education. Pediatric ED data generated through our electronic medical record as well as through Medicaid reimbursement data framed the scope of this issue as significant. This was used to monitor pediatric ED visits following interventions as well.

Results: Over the initial 3 months of interventions, pediatric ED use decreased by 62 visits compared with the prior year. The low-acuity diagnoses of upper respiratory infections decreased by $43.7 \%$ ( 71 to 40 visits) and fever decreased by $50.0 \%$ (14 to 7 visits) from the same 3 months the year prior. This decrease was sustained when examined during year 3. Over the next 12 months, there were 284 $\mathbf{2 9 . 8 \% )}$ less visits to the ED with low-acuity diagnoses. This calculates to approximately $\$ 300,000$ saved to the Medicaid system. During this time frame, our pediatric panel increased by 200 patients.

Discussion: Increasing access and improving patient education decreased low-acuity pediatric ED visits in our clinic. This combination of interventions worked well in our community and has been shown to help optimize the setting in which pediatric patients are seen. (J Am Board Fam Med 2018;31: 550-557.)

Keywords: Ambulatory Care Facilities, Child, Family Medicine, Hospital Emergency Service, Pediatrics, Surveys and Questionnaires

Patient overuse of the emergency department (ED) has been a long-standing issue in all medical communities. The family medicine residency clinic at

\footnotetext{
This article was externally peer reviewed.

Submitted 28 December 2017; revised 12 March 2018; accepted 13 March 2018.

From New Hanover Regional Medical Center Residency

in Family Medicine, Wilmington, NC (TD, AM, JB, SB).

Funding: none.

Conflict of interest: none declared.

Corresponding author: Toren Davis, DO, New Hanover Regional Medical Center Residency in Family Medicine, 2532 Delaney Avenue, Wilmington NC, 28403 (E-mail: Toren.davis@NHRMC.org).
}

New Hanover Regional Medical Center cares for a large number of the community's children with Medicaid coverage, as there are very few clinics in the area that accept new Medicaid pediatric patients. Multiple studies have shown that patients with Medicaid are the most frequent visitors to the $\mathrm{ED}^{1}$. According to the Centers for Disease Control and Prevention's 2012 National Center for Health Statistics Report, children with Medicaid used the $\mathrm{ED}$ at a rate of about $25 \%$, whereas uninsured children used the ED at $16 \%$, and children with private insurance used it at $13 \%^{2}$. Over the past 3 years, the issue of Medicaid accessibility for pa- 
tients in our community has led to our pediatric patient population increasing from 22\% (1078 patients) of our total panel to $32 \%$ (1414 patients), with $88 \%$ of those patients using Medicaid as their main form of payment, which was shown from multiple analyses of our electronic medical record reports. As an unintended consequence of this significant increase, the issue of ED overuse for lowacuity visits became an even more prominent issue for our clinic. The low acuity of these patients is indicated by the fact that $95.5 \%$ are "treat and release," meaning they do not require hospitalization $^{3}$. The most common pediatric ED diagnoses are upper respiratory infection (URI), fever, and otitis media, all well within the scope of an ambulatory setting ${ }^{4}$. National ED frequency data further indicates that the highest pediatric users of the ED are in the 0 years to 3 years age range ${ }^{5}$. This age group is where our clinic has seen the greatest increase in pediatric patients over the past 2 years.

Bringing pediatric patients with low-acuity health issues to their primary care facility instead of the ED has many benefits. One study showed that the primary care setting provides better continuity, less unnecessary or duplicative testing, decreased wait time, and lowers the burden on an already strained Medicaid system ${ }^{6,7}$. The addition of valuebased reimbursement also adds to the importance of seeing pediatric patients in an ambulatory setting to increase cost savings for both the patient and the care provider.

As shown previously by the Health Care Cost and Utilization Project, some of the most common barriers to care for a primary care facility, thus leading to an increase in avoidable ED use, were greater distance to the office versus the ED, lack of availability for sick appointments, lack of nurse triage line availability, lack of acceptance of all sick walk-in visits, and lack of same-day turnaround $\mathrm{labs}^{8}$. Parental education has also been shown to be a determining factor for medical decision making? One solution to the parental education barrier from a 2011 Texas A\&M University study gave parents handbooks that discussed common pediatric illnesses. The study resulted in a decrease in lowacuity visits to the $\mathrm{ED}^{10}$. Another successful decrease of low-acuity pediatric ED use was seen by using a health book to educate parents on pediatric illnesses ${ }^{11}$. The combination of these barriers, among others, attribute to the high number of avoidable low-acuity ED visits. Although the extent of this issue varies by medical community, an emphasis on patient-identified barriers to care from our community was taken to maximize potential benefits from our interventions. We hypothesize that improving same-day access to the clinic alongside improved parental education will further decrease low-acuity ED visits.

\section{Methods}

A survey was sent to 25 parents whose children were seen in the ED more than 2 times in the previous 6 months to discern why they chose the ED over the clinic (Table 1). This method was chosen to find patient-identified barriers to care that kept patients from using our clinic over the ED. The most common answer recorded was that parents were not aware of what the clinic offered in regard to acute service. However, if they were

Table 1. Questionnaire with Responses for High-Use Emergency Department Pediatric Patients

\begin{tabular}{|c|c|c|}
\hline Question & n (\%); Response & Total Responses \\
\hline $\begin{array}{l}\text { 1. Were you discharged home from the } \\
\text { emergency room? }\end{array}$ & 25 (100); Yes & 25 \\
\hline $\begin{array}{l}\text { 2. Were you aware of the Coastal Family } \\
\text { Medicine on-call line? }\end{array}$ & 5 (20); Yes & 25 \\
\hline $\begin{array}{l}\text { 3. If yes, did you call the Coastal Family } \\
\text { Medicine on-call line? }\end{array}$ & $1(4) ;$ Yes & 25 \\
\hline $\begin{array}{l}\text { 4. If during business hours, did you attempt to } \\
\text { call the clinic for an acute appointment? }\end{array}$ & 3 (12); Yes & 25 \\
\hline \multirow{3}{*}{$\begin{array}{l}\text { 5. If you used the emergency department, why } \\
\text { did you choose that over Coastal Family } \\
\text { Medicine? }\end{array}$} & $\begin{array}{l}10 \text { (40); emergency department easier than using } \\
\text { clinic. }\end{array}$ & \multirow[t]{3}{*}{25} \\
\hline & $\begin{array}{l}12 \text { (48); were not aware of outpatient acute } \\
\text { services. }\end{array}$ & \\
\hline & $\begin{array}{l}3 \text { (12); did not think the clinic could handle the } \\
\text { child's issue. }\end{array}$ & \\
\hline
\end{tabular}


aware, they went to the ED because it was easier than accessing the clinic (Table 1). A review of the current literature yielded similar results to barriers to care across the country. There were multiple studies showing that increased access to the outpatient clinic as well as improved parent education were important to decreasing unnecessary ED use. The methods used to increase access included those such as increasing walk-in appointment numbers, adding evening hours, and adding weekend hours. The attempts at improved parent and patient education ranged widely, with examples including parent handouts, more dedicated time for discussion in the examination room, and increased access to a triage line ${ }^{12}$. In addition to reviewing the literature, other practices in our community serving the pediatric population were examined to assess their differences in providing outpatient care. The common denominator and difference from our clinic when examining these 6 other practices was widerreaching same-day services. Using this data to establish current barriers to care and patient needs in conjunction with recent literature on the subject, our quality team, which consists of front desk and rooming staff, nurses, resident physicians, attending physicians, and our practice manager, met and established guidelines to improve access.

At these meetings, our prior procedures were examined and found to not optimally meet our patient's needs in regard to access to care and parent education. The clinic policy for seeing acute same-day pediatric patients was standardized, and a walk-in clinic for ages 0 years to 18 years was created during business hours, Monday through Friday. To improve parent education, posters describing our walk-in clinic were placed in the waiting area as well as examination rooms, office staff and physicians were given updated scripting to discuss with patients, the after-hours phone triage line was updated, and bookmarks that described our walk-in clinic as well as an after-hours call line were placed in the books our children get at each wellchild examination. We chose these interventions to pursue because they fit the areas our patients identified as needing improvement, could be implemented with our current clinic infrastructure, and had evidence of some success in other populations on review of the literature.

The definition of a low-acuity visit in this study took into account our organization's current acuity scale used to prioritize acuity in the ED. On arrival, each patient is assessed using the Emergency Severity Index. This score gives a patient a score of 1 to 5: a score of 1 needing immediate attention, 2 being emergent, 3 being urgent, 4 being less urgent, and 5 being not urgent ${ }^{13}$. By examining the criteria in each of these acuity levels, we found that levels 3 through 5 would, in most cases, be appropriate for an outpatient setting. Level 1 and 2 would most appropriately be seen within the ED.

An electronic medical record-created report of ED use was run each month retroactively for the year prior and also generated monthly for 1 year following interventions. Acuity level, time of ED visit, age, primary provider, and visit diagnosis were examined with the report. This was compared with Medicaid reimbursement data for our patients. The project was reviewed through our institutional review board and granted exemption.

\section{Results}

Despite the fact that the pediatric panel grew $17.5 \%$ (1203 to 1414 patients), we saw 62 less pediatric ED visits than the same 3 months the year prior within 3 months of using our new procedures. There was a noticeable shift and decrease in pediatric use of the ED following the July 2017 initiation of interventions (Figure 1). From that point on, each month postintervention saw less visits than the corresponding month preintervention. The numbers continued to decrease 12 months after initiation of our interventions. There was a $284-$ visit decrease in total pediatric $\mathrm{ED}$ visits $(29.8 \%)$ from our clinic 12 months after interventions started. This roughly saved $\$ 300,000$ for the Medicaid system in that time when compared with the year prior. There was a $41.1 \%$ (82 to 48 ) decrease in level 3 (urgent) visits to the ED and a 16.7\% (120 to 100) decrease in level 4 (less urgent) visits in the initial 3 months (Figure 2). These changes were maintained when examining the data a year later, from October-December 2017. The level 3 (acute) visit decrease was found to be statistically significant $(P=.0019)$. Emergent acuity-level visits to the ED remained unchanged from the year prior. Level 5 (not acute) visits also remained unchanged through years examined. On the level of diagnosis, there was a $29.0 \%$ decrease in URI diagnosis (71 to 40 visits) and a $50.0 \%$ decrease in fever diagnosis (14 to 7 visits) from the year prior during the initial 3 months. These changes, as well, were maintained 
Figure 1. Time line map of interventions, with associated change in pediatric emergency department (ED) visit volume. Postintervention data show a decrease in total ED visits below that of preintervention at approximately the same time interventions started.

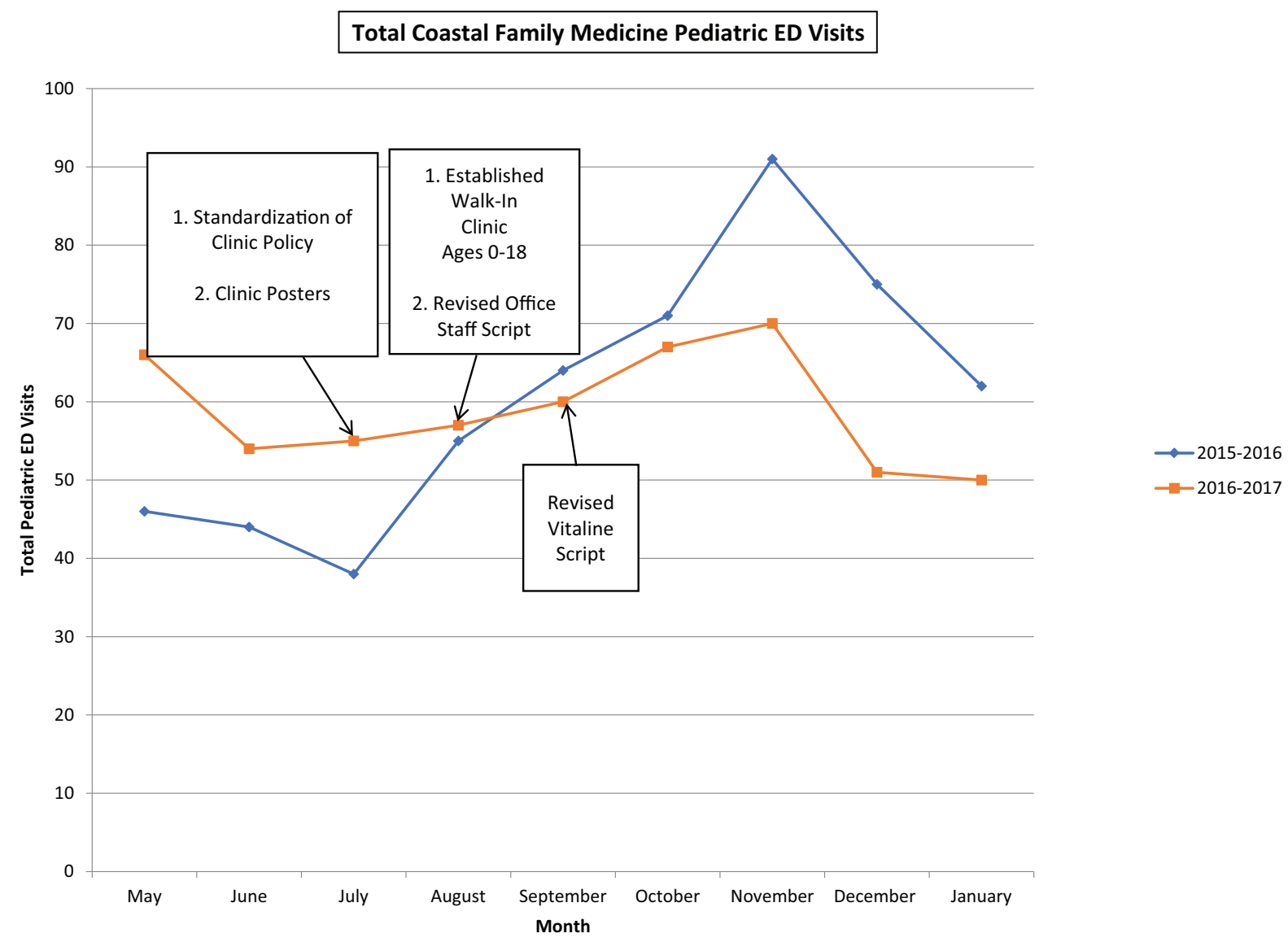

after a year when looking at the October-December 2017 data.

Looking at the national data for pediatric panel size and ED use, we found that our study showed improvement as well. The 2014 data shown by the National Hospital Ambulatory Medical Care Survey showed the number of ED visits per 100 persons per year for pediatric patients to be $45.5^{14}$. In 2015 , our clinic was at 71.5 visits per 100 persons per year. Following interventions, in 2017, our rate changed to 41.9 visits per 100 persons per year.

Walk-in clinic visits during this time continued to increase. In the early months of the walk-in clinic, before adequate education of patients and parents, there was an average of 20 to 30 pediatric walk-in visits. One year after continued education to our patient panel, the walk-in clinic saw 120 pediatric walk-in visits in September 2017 and 132 visits in October of 2017 (Figure 3). The propor- tions of our pediatric patients using our clinic instead of the ED also shifted from 2015 to 2017. In the October-December time frame, we saw $47.5 \%$ (64 patients) of our patients use the clinic for URI in 2015 . In 2017, that number increased to $78.2 \%$ (97 patients) that were seen in the clinic for a diagnosis of URI as opposed to the ED. This was a statistically significant shift $(P<.0001)$ (Figure 4). Similar shifts were seen in fever and otitis media; however, the sample size was not great enough to draw significance conclusions. The increased use of the walk-in clinic corresponded with the continued decrease in total pediatric ED visits as the panel size grew by over 200 patients.

\section{Discussion}

The standardized changes in our clinic access policies and use of walk-in sick child slots during business hours have improved outpatient access. 
Figure 2. Emergency department (ED) visit acuity before and after interventions. Low acuity level 3 (urgent) saw a statistically significant decrease from 2015 to 2016 and maintained the decrease into 2017. Level 4 (less urgent) saw a decrease that continued in 2017 but was not statistically significant. Emergent (level 2) visits were unchanged throughout. Emergency Severity Index Scale used for acuity scoring (1, immediate; 2, emergent; 3, urgent; 4 , less urgent; 5 , not urgent) designated level 3 to 5 as low acuity.

October-December Pediatric ED Visit Acuity

140

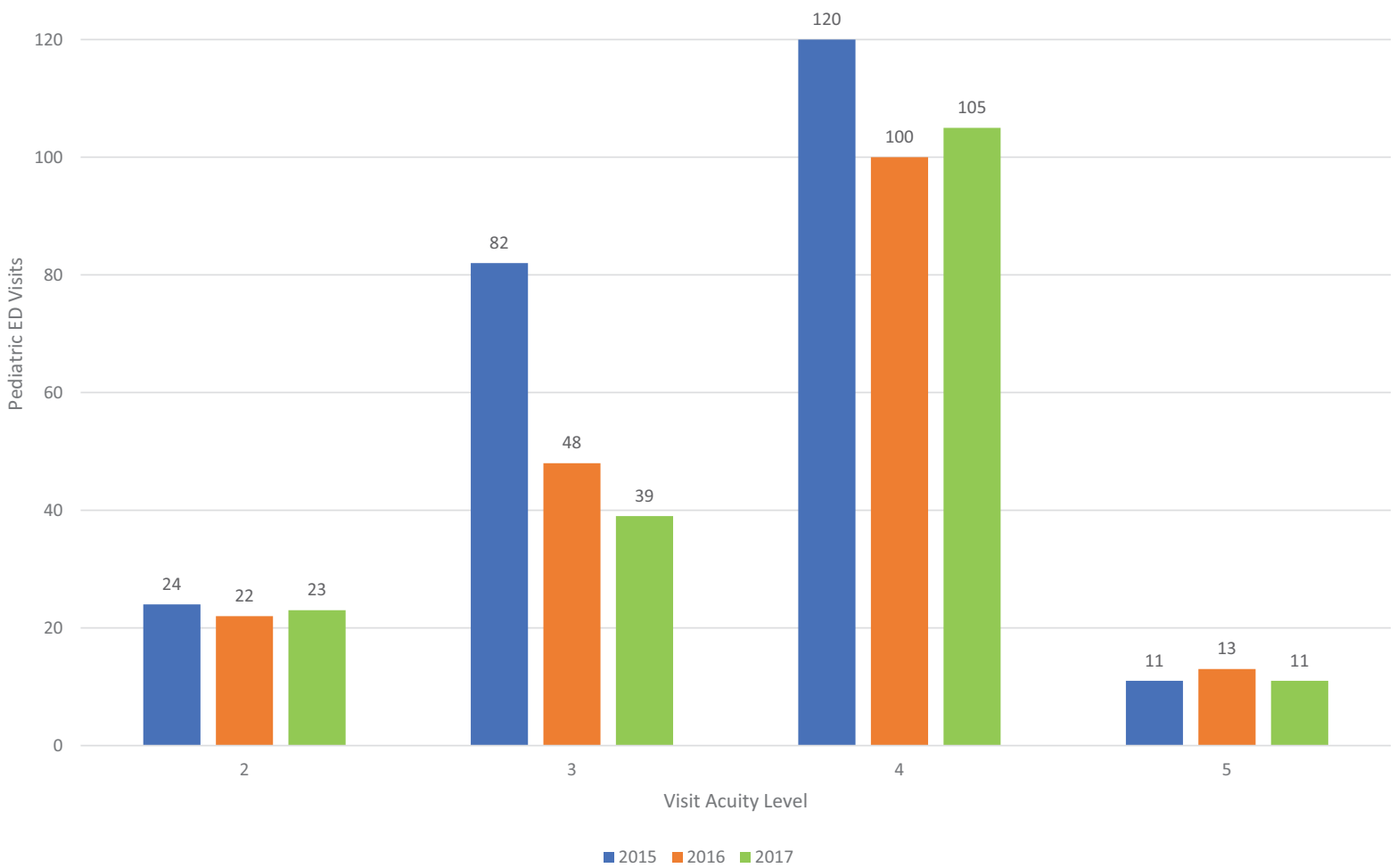

In conjuncture with increased parent education, we saw a decrease in low-acuity ED use. This was noticeable through the increased walk in clinic use and decrease in ED visits by our pediatric population. By making changes to eliminate patient-identified barriers to care, we noted a change despite the increase in panel size by $17.5 \%$ from the year prior. With the amount of low-acuity ED visits decreasing as the emergent ED visits remaining the same as the year prior, our interventions successfully altered low-acuity visits. Continued parent and patient education concerning the appropriate use of the ED and the clinic, along with our increase in accessibility to immediate appointments, gave parents the tools they needed to decide where their child's needs were best met.

Although every clinic and community has variations in barriers to care, the method of evaluating these barriers and developing a protocol for issues such as walk-in availability can be considered elsewhere. By addressing the common concerns from our patient community and focusing our energy in these areas, our study demonstrated that increasing the accessibility to the clinic and improving parent education were key to decreasing low-acuity ED use in our area.

Although our study determined a $29.8 \%$ decrease in pediatric ED use over the last year, most of our increase in access occurred during business hours. Prior studies established that approximately $40 \%$ of pediatric ED visits occur after business hours, ${ }^{15,16}$ between the hours of 5 PM- 8 AM and during the weekend. Expanding clinic hours to reach these visits is a logical next step. An increase in access during business hours coupled with improved parent education yielded the results we found. It is not beyond reason to hypothesize that 
Figure 3. Pediatric emergency department (ED) visits from the year before interventions and after interventions. Pediatric walk-in clinic use is also depicted here with the blue line. The ED use numbers were lower than the year prior for every month since starting our changes, while the walk-in clinic use continues to increase.

Pediatric Emergency Room Visits and Walk in Clinic Utilization

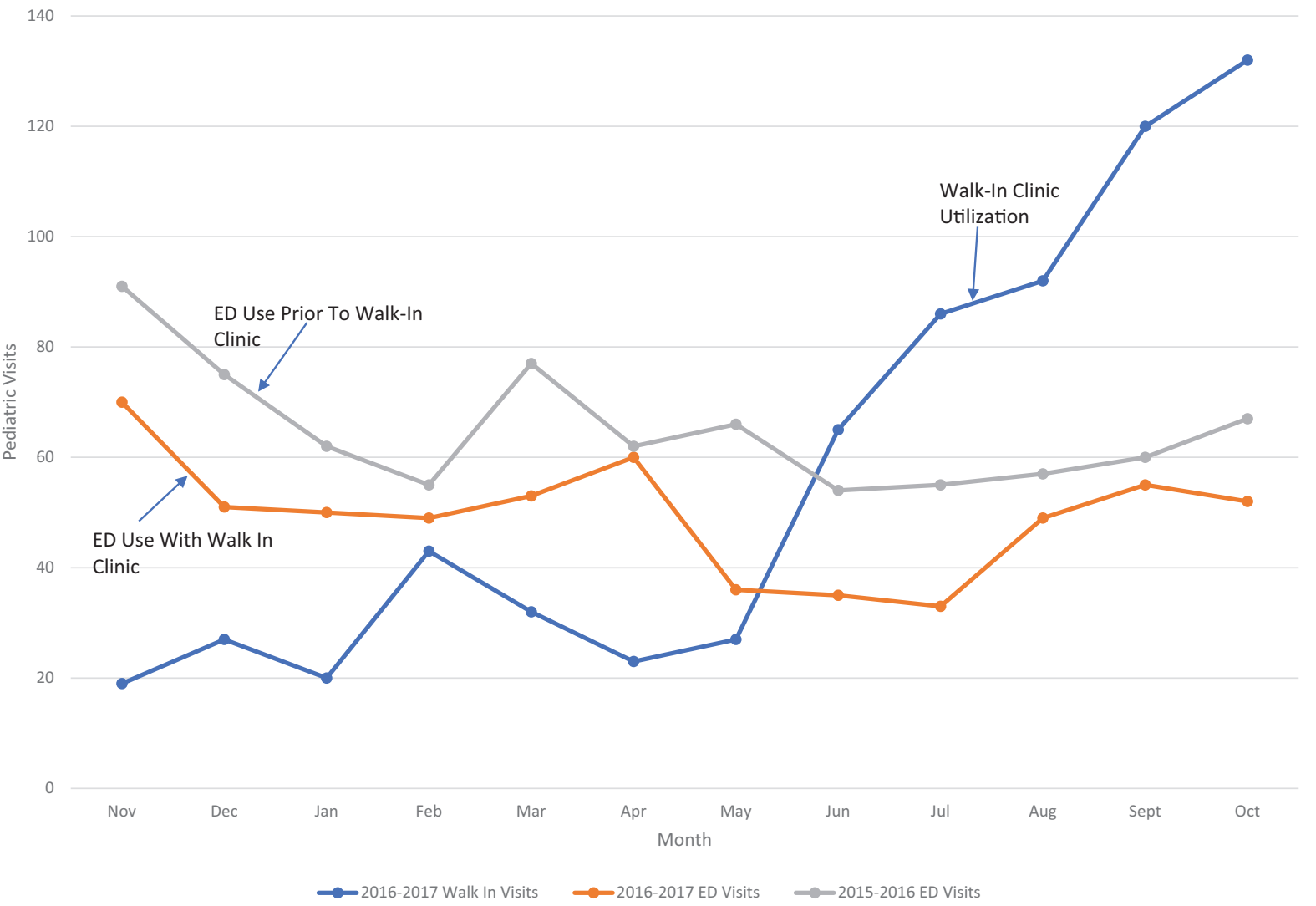

adding after-hour access to our clinic in connection to the improved parent education will achieve further decreases in low-acuity ED visits. Evening hours captured a large portion of these visits, especially with families where the parents work during the day. ${ }^{16}$ This is one area we plan to go in the future, following further patient-centered survey results.

As the future points toward value-based reimbursement, the redirection of patients to appropriate levels of care will gain importance within the medical community. As family physicians, we are trained and equipped to care for many of the chief complaints that make it to the ED each day. The patients are seen by their provider and continuity is preserved, which is a service that builds on the foundation of family medicine. It is engrained in us during our training and a main component of the care we provide. By focusing on patient-centered interventions and eliminating barriers to care, the changes we implemented can be replicated in other medical communities with ease.

The study design did have limitations. The limits in sample size of our single clinic made it difficult to deduce statistical significance from some of our changes. However, statistical significance was found in terms of reduced less-acute (level 3) ED visits as well as the shift in patients seen in the clinic versus the ED for URI. The small sample size for fever and otitis media was a weakness. There were similar shifts seen to URI; however, the sample size did not yield statistical significance. Yearly changes in acute illnesses such as influenza could also act as a confounder. By examining our 3-month period, October-December, for 3 consecutive years 2015 to 2017 , we showed a maintained change from our baseline data, even with flu seasons of differing intensities. Our study only changed walk-in access during business hours. On further examination of our acuity data, $75.0 \%$ (75 visits) of the level 4 (less 
Figure 4. Shifts in patients seen in the emergency department (ED) versus the clinic before interventions (2015) and after interventions (2017). Upper respiratory infections went from $47.5 \%$ seen in the clinic to $78.2 \%$ in 2017 . This was statistically significant $(P<.0001)$. Both fever and otitis media showed similar changes, but the small sample size made them not statistically significant (fever, $P=.078$ ).

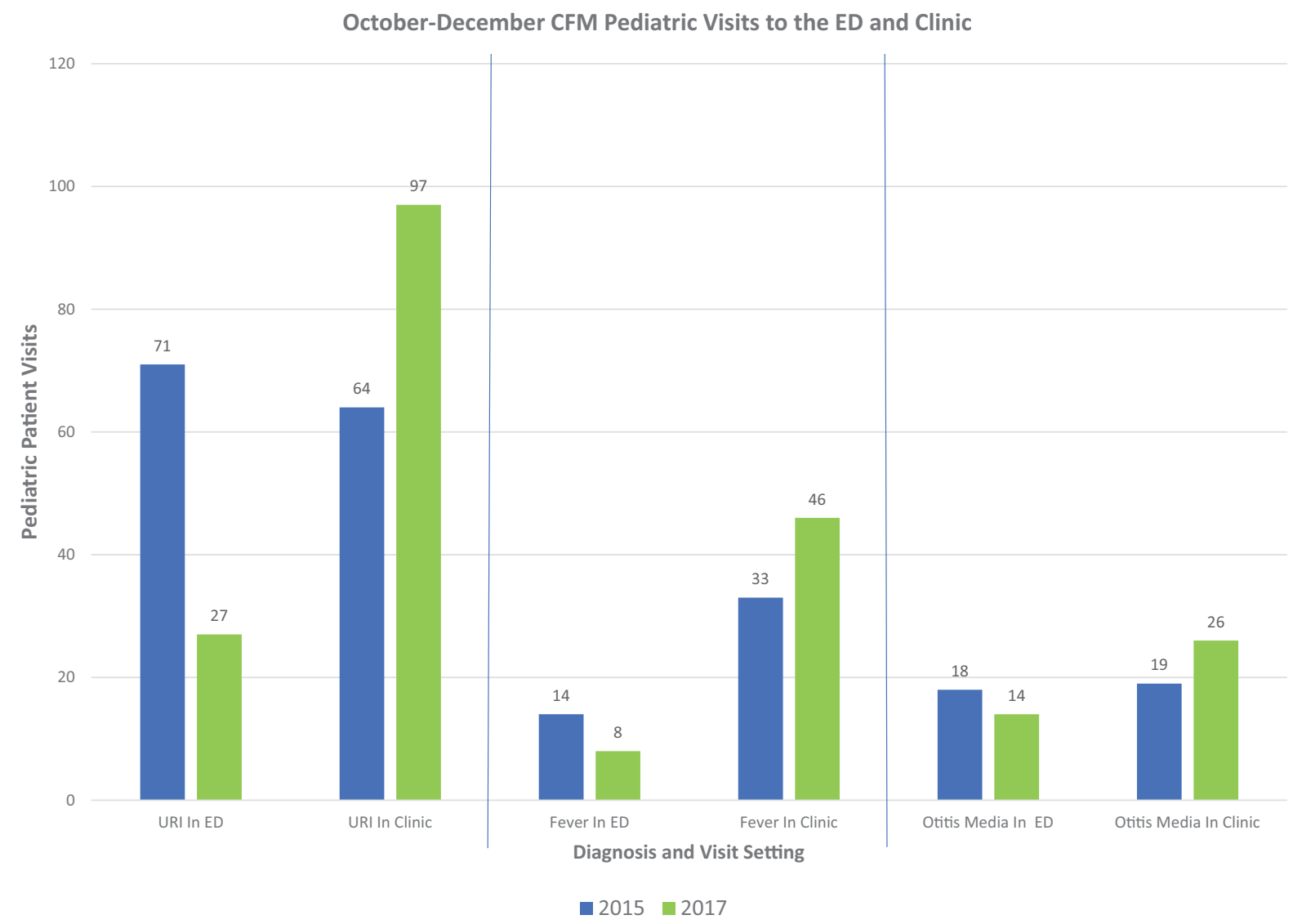

acute) and $90.1 \%$ (10 visits) of level 5 (not acute) pediatric visits were noted to be after business hours or during the weekend. This could somewhat explain why our level 4 (less acute) visits did not decrease in the same amount that our level 3 (acute) visits did. Patients seen during these times would not benefit from our increased access during business hours, as noted above. This would be a good area for further examination.

The authors acknowledge Caroline Devries for contributing to the editing process.

To see this article online, please go to: bttp://jabfm.org/content/ 31/4/550.full.

\section{References}

1. Wier LM, Yu H, Owens PL, Washington R. Overview of children in the emergency department, 2010. Rockville (MD): Healthcare Cost and Utilization Project; 2013:157.
2. Reasons for Emergency Room Use Among US Children: National Health Interview Survey. CDC National Center for Health Statistics Report: NCHS Data Brief No. 160; 2012. Available from: https:// www.cdc.gov/nchs/data/databriefs/db160.htm.

3. Merrill CT, Owens PL, Stocks C. Pediatric emergency department visits in community hospitals from selected states, 2005. Rockville (MD): Healthcare Cost and Utilization Project (HCUP) Statistical Briefs; 2008:52.

4. Health, United States, 2015: With Special Focus on Racial and Ethnic Health Disparities. Hyattsville (MD): U.S. Department of Health and Human Services; 2015:270-4.

5. Grossman LK, Rich LN, Johnson C. Decreasing non-urgent emergency department utilization by Medicaid children. Pediatrics 1998;102:20-4.

6. Alpern ER, Clark AE, Alessandrini EA, et al. Recurrent and high-frequency use of the emergency department by pediatric patients. Acad Emerg Med 2014;21:365-73. 
7. McBurney PG, Simpson KN, Darden PM. Potential cost savings of decreased emergency department visits through increased continuity in a pediatric medical home. Ambul Pediatr 2004;4:204-8.

8. Center for Delivery, Organization, and Markets, Healthcare Cost and Utilization Project (HCUP), Nationwide Emergency Department Sample (NEDS). Rockville, MD: Agency for Healthcare Research and Quality (AHRQ); Statistical Brief No. 157. 2010. Available from: https://www.ncbi.nlm.nih.gov/ books/NBK154386/.

9. Kubicek $\mathrm{K}$ et al. A profile of non-urgent emergency department use in an urban pediatric hospital. Pediatr Emerg Care 2012;977-84.

10. Yoffe SJ, et al. A reduction in emergency department use by children from a parent educational intervention. Fam Med 2011;43:106-11.

11. Hernan A, Jackson. Empowering low-income parents with skills to reduce excess pediatric emergency room and clinic visits through a tailored low literacy training. J Health Comm 2010;895-910.
12. Morgan SR, Chang AM, Algatari M, Pines JM. Non-emergency (ED) interventions to reduce ED utilization: a systemic review. Acad Emerg Med. 2013;20:969-985.

13. Gilboy N, Tanabe P, Travers D, Rosenau A. Emergency Severity Index (ESI): a research based triage tool for emergency department care, version 4. AHRQ Publications No. 12-0014. Rockville (MD): Agency for Healthcare Research and Quality; 2012.

14. National Hospital Ambulatory Med Care Survey. Atlanta (GA): Centers for Disease Control and Prevention; 2014.

15. Young GP, Warner MB, Kellerman AL, Ellis J, Bouley D. Ambulatory visits to hospital emergency departments: patterns and reasons for use. JAMA 1996;276:460-5.

16. Piehl MD, Clemens CJ, Joinesw JD. Narrowing the gap: decreasing emergency department use by children enrolled in the Medicaid program by improving access to primary care. Arch Pediatr Adolesc Med 2000;154:791-5. 